\title{
Implementación de un Modelo de Capacitación para Docentes de Bachillerato, por Medio de Entornos Virtuales de Aprendizaje, una Respuesta ante la Pandemia
}

\author{
Implementation of a Training Model for High School Teachers, Through Virtual \\ Learning Environments, a Response to the Pandemic
}

Jahzeel Quintanilla ${ }^{1}$ y Minerva Castillo ${ }^{2}$

\section{ఠ} EDICIÓN: 进e-CIVTAC

Recibido: 19/julio/2020

Aceptado: 12/agosto/2020

Publicado: 25/septiembre/2020

我 México

2México

\section{IIIIInstitución}

${ }^{1}$ CONALEP de Nuevo León

${ }^{2}$ Universidad Ciudadana de Nuevo León

\section{Correo Eletrónico}

1j_quintanilla@conalepnl.edu.mx ²minerva.castillo@ucnl.edu.mx

\section{DORCID}

1https://orcid.org/0000-0003-2883-9556 ${ }^{2 h t t p s: / / o r c i d . o r g / 0000-0001-8301-2426 ~}$

\section{Citar así: CCAPA / IEEE}

Quintanilla, J. \& Castillo, M. (2020). Implementación de un Modelo de Capacitación para Docentes de Bachillerato, por Medio de Entornos Virtuales de Aprendizaje, una Respuesta ante la Pandemia. Revista TecnológicaEducativa Docentes 2.0, 9(2), 182-191. https://doi.org/10.37843/rted.v9i2.163

J. Quintanilla y M. Castillo, "Implementación de un Modelo de Capacitación para Docentes de Bachillerato, por Medio de Entornos Virtuales de Aprendizaje, una Respuesta ante la Pandemia", RTED, vol. 9, n. ${ }^{\circ}$, pp. 182-191, sep. 2020.

\section{Resumen}

La presente investigación refiere el trabajo realizado con docentes del nivel bachillerato en México, inicia con la declaración de emergencia por pandemia del COVID-19, por parte de la Organización Mundial de la Salud (2019), citado en el Diario Oficial de la Federación (DOF, 2020) en consecuencia, el Gobierno de México establece medidas de confinamiento para preservar la salud poblacional, en todas las escuelas del país, se interrumpen las clases presenciales en el Colegio de Educación Profesional Técnica en el Estado de Nuevo León (Conalep Nuevo León), bajo este contexto, ésta investigación tiene como finalidad: implementar un modelo de capacitación a docentes de Conalep Nuevo León, en modalidad en línea, para adquirir habilidades digitales en el desarrollo y construcción de un aula virtual. Se optó por una metodología cuantitativa de tipo exploratoria, descriptiva, la muestra estuvo compuesta por 66 docentes representantes de los 17 planteles, designados por parte de la dirección académica institucional. Para el análisis se consideró el uso de herramientas estadísticas, los datos obtenidos confirman la consolidación del objetivo planteado, con una aprobación del $88 \%$, el trabajo realizado por los docentes, se dividió en aspectos teóricos y prácticos, dentro de los principales hallazgos se evidencia la necesidad de reforzar la ejecución y construcción de los elementos en plataforma, así mismo se identifica el grupo de mayor edad de los docentes, como quienes presentaron dificultades en la realización, se identifican sus procesos de ejecución en línea, así como los vacíos de conocimiento respecto al trabajo en entornos virtuales de aprendizaje.

Palabras clave: EVA, Docentes, Habilidades digitales, Bachillerato.

\section{Abstract}

This research refers to the work carried out with high school teachers in Mexico, it begins with the declaration of pandemic emergency of COVID-19, by the World Health Organization (2019), quoted in the Official Gazette of the Federation ( DOF) consequently, the Government of Mexico establishes confinement measures to preserve population health, in all schools in the country, face-to-face classes are interrupted at the College of Professional Technical Education in the State of Nuevo León (Conalep Nuevo León), In this context, this research aims to: implement a training model for teachers in Conalep Nuevo León, online, to acquire digital skills in the development and construction of a virtual classroom. An exploratory, descriptive quantitative methodology was chosen, the sample was made up of 66 teachers representing the 17 schools, appointed by the institutional academic management. For the analysis, the use of statistical tools was considered, the data obtained confirms the consolidation of the proposed objective, with an approval of $88 \%$, the work carried out by the teachers was divided into theoretical and practical aspects, among the main findings is evidence the need to reinforce the execution and construction of the elements on the platform, likewise the oldest group of teachers is identified, as those who presented difficulties in the realization, their online execution processes are identified, as well as knowledge gaps regarding work in virtual learning environments.

Keywords: High School, teachers, EVA, digital skills, pandemic. 
Implementación de un Modelo de Capacitación para Docentes de Bachillerato, por Medio de Entornos Virtuales de Aprendizaje, una Respuesta ante la Pandemia.

\section{Introducción}

Ante la declaración del COVID-19 como pandemia por parte de la Organización Mundial de la Salud (OMS) el pasado 11 de marzo del 2020, se emitió una emergencia en el área de Salud Pública con repercusión a nivel Internacional, en México las Secretarías de Salud y de Educación Pública, el 14 de marzo del 2020, presentaron diversas medidas preventivas, así como su atención prioritaria en seguimiento a las recomendaciones implementadas por la OMS, enfocadas en contribuir a preservar la salud de niñas, niños, adolescentes y jóvenes, así como del resto de la comunidad en todos los planteles educativos del país. (DOF, 2020)

A propósito del efecto en el ámbito educativo, en resolución a esta pandemia del COVID-19 se ha requerido el cierre de escuelas a nivel mundial, la Organización de las Naciones Unidas, destaca en muchos países se enfrentan a una importante interrupción en la enseñanza causada por el COVID19, por su parte la Organización de las Naciones Unidas para la Educación, la Ciencia y la Cultura, presentó el documento sobre Coalición Mundial para la Educación con el objetivo de ofrecer a los niños y jóvenes opciones referentes al aprendizaje inclusivo durante este período de interrupción repentina, sin precedentes en la educación. (UNESCO, 2020)

Las disposiciones sanitarias dadas a conocer por la Secretaría de Salud en México, llevaron al Colegio Nacional de Educación Profesional Técnica (CONALEP), a suspender clases a partir del 18 de marzo del 2020, resultando necesario tomar medidas de acción con los docentes del plantel, dedicados a impartir clases presenciales; el perfil docente en CONALEP, define las características del profesional en educación requerido, considerando tanto el análisis para su función, el proceso en solución de problemas, así como el reconocimiento de sus funciones cotidianas.

Es importante mencionar, además, el reto de los docentes en el sistema CONALEP incluye atender población de alto riesgo, diversificación cultural de los alumnos, grupos heterogéneos, inequidad en el acceso al conocimiento y saberes en permanente evolución disciplinar, cultural, laboral y social, por lo tanto, debe estar preparado y mantenerse en constante actualización. (Ibarra, 2019, párrafo 3).

Actualmente Conalep Nuevo León, se apega al modelo académico 2018 definido por Conalep
Nuevo León, se apega al modelo académico 2018 definido por Conalep México, respetando su estructura en los programas de estudio, la guía pedagógica, así como la forma en evaluar a los alumnos, por medio de rúbricas ya diseñadas, ofreciendo una evaluación flexible, formativa y permanente. Ante este contexto de cierre institucional, y el compromiso de seguir con los objetivos académicos institucionales, surgen las siguientes interrogantes: ¿Cómo seguir impartiendo el proceso de enseñanza, sin clases presenciales? ¿Cómo migrar al docente presencial al contexto virtual?

Las Naciones Unidas, por medio del departamento de comunicación global (2020), menciona que, mientras los países se enfrentan a una importante interrupción en la enseñanza causada por el COVID-19, la UNESCO ha hecho un llamamiento para facilitar el aprendizaje y un intercambio en conocimientos mediante recursos educativos abiertos. Siendo estos, materiales didácticos, de aprendizaje o investigación - en cualquier formato o medio - que se encuentren en el dominio público o que se publiquen con licencias de propiedad intelectual que faciliten su uso, adaptación y distribución gratuitos. (UN, 2020)

La educación virtual se ha hecho popular debido a su potencial para proveer acceso flexible a los contenidos, instrucción en cualquier tiempo, así como desde cualquier lugar a través de diversas aplicaciones tecnológicas las cuales permiten comunicación asincrónica y sincrónica tal como señalan, Arras, Valencia \& Tejedor (2014, p.3). Complementando, el uso de la tecnología es una nueva manera en gestionar el conocimiento cuya finalidad primordial es crear un entorno apropiado para enseñar y aplicar con éxito este proceso, requiere de métodos pedagógicos adecuados con respuestas favorables a las nuevas necesidades que imponen las innovaciones rápidas, tal como expresan Betegón, Fossas, Martínez \& Ramos (2010, p. 277).

Derivado de la emergencia de salud pública, un grupo de expertos de Conalep Nuevo León, identifican el apuro para fortalecer en los docentes las habilidades digitales necesarias, y realizar las funciones a distancia, mediante un modelo de capacitación para diseñar e impartir clases en línea. El reto consistió en migrar de ser un docente en un aula presencial con horarios establecidos y grupos de estudiantes definidos, a ser un facilitador aplicando habilidades digitales en la construcción y diseño de 
Implementación de un Modelo de Capacitación para Docentes de Bachillerato, por Medio de Entornos Virtuales de Aprendizaje, una Respuesta ante la Pandemia. aulas virtuales.

En este sentido, es relevante desarrollar un modelo de capacitación docente cuyo fin sea cumplir con los elementos básicos de habilidades digitales sobre diseñar, construir e interactuar en el manejo de aulas virtuales, para favorecer el desarrollo de competencias en la incorporación de las TIC en sus procesos de enseñanza, por lo tanto, la presente investigación tuvo como objetivo general: Implementar un modelo de capacitación a docentes de Conalep Nuevo León en modalidad en línea, para adquirir conocimientos en habilidades digitales en el desarrollo y construcción de un aula virtual.

Se requiere pasar del diseño en educación presencial, al diseño con formato educativo virtual, en este sentido, el rol cambia de un docente en el aula, a ser un facilitador del proceso enseñanzaaprendizaje en línea. En seguimiento a lo anterior, Betegón, Fossas, Martínez \& Ramos (2010, p.279), establecen las siguientes características de la educación virtual: el profesor puede realizar un seguimiento continuado en los alumnos, mediante los resultados de ejercicios, test de autoevaluación y participación para los alumnos a través de diversas herramientas de comunicación, lo cual es uno de los pilares fundamentales en los entornos de enseñanzaaprendizaje virtuales, pues permite el diálogo muto entre todas las personas implicadas mediante diferentes herramientas como gestores de correo, aplicaciones, chat, entre otros.

Los mismos autores mencionan respecto al trabajo colaborativo, que éste se favorece en los entornos virtuales de aprendizaje, mediante el uso de diferentes aplicaciones mismas que sirvan para distribuir información, trabajar de manera conjunta y favorecer la resolución de problemas, en seguimiento a la idea, manifiestan que los entornos virtuales permiten y favorecen los procesos de gestión académica y administración de los alumnos, así mismo posibilitan la consulta de los expedientes de los alumnos. (Betegón, Fossas, Martínez \& Ramos, 2010, p.279).

Para finalizar los autores mencionan que dentro de las ventajas en los entornos virtuales se encuentra el proporcionar acceso inmediato a diversos elementos de aprendizaje, como son las simulaciones, archivos, imágenes, entre otros, enfatizan que los entornos virtuales, favorecen y promueven la interacción en cuatro niveles: alumnoprofesor, proporcionando motivación, feedback, entre profesor-alumno; alumno-contenido, donde el alumno recibe información intelectual del material; alumno-alumno, pues se produce intercambio de información, ideas, diálogo, por último, alumnointerfaz, es decir, entre el alumno y la tecnología, transmisora de información. (Betegón, Fossas Martínez \& Ramos,2010 p.280).

Landázuri (2017) refiere que muchas instituciones educativas, emplean programas de educación online y combinan tecnología con educación para impartir o producir material actualizado, afirma que el aprendizaje necesita más de tecnología, la cual constituye un medio por el cual comparten contenidos, además realizan evaluaciones de conocimiento, en consecuencia, el implementar educación virtual, promueve la flexibilidad y el acceso a la información permitiendo la inclusión de diversos materiales, responde de manera directa a la necesidad de evitar reuniones o aglomeraciones, derivadas de la pandemia y favorece el proceso de interacción entre los miembros del grupo.

En este sentido el Colegio Nacional de Educación Profesional Técnica (CONALEP) intensificó los trabajos del programa de educación a distancia, con objeto de garantizar una formación constante en sus estudiantes y docentes ante contingencias como la que atraviesa el país a causa del COVID-19 (Secretaría de Educación Pública, 2020). Conalep Nuevo León, cuenta con 17 planteles en el estado con una matrícula de más de 21 mil estudiantes, 1,048 miembros del personal docente, contratados en el periodo semestral febrero a julio de 2020.

Como se ha mencionado previamente los docentes, responden al modelo académico de la institución, (Conalep, 2011), cuya práctica cotidiana se realiza frente a grupo en las aulas del colegio, son contratados para impartir clases presenciales, a un máximo de 20 horas clase por semana de acuerdo con el turno matutino o vespertino. Con la necesidad del desarrollo de habilidades digitales en los docentes, es pertinente su inserción en los entornos virtuales de aprendizaje, los cuales constituyen una herramienta para formar en línea e ir transformado los métodos de aprendizaje de manera vertiginosa al eliminar las delimitaciones de espacio y tiempo en el proceso formativo.

Por su parte Salinas (2011 p.1), puntualiza que, un entorno virtual de aprendizaje (EVA) es un espacio educativo alojado en la web, conformado por un 
Implementación de un Modelo de Capacitación para Docentes de Bachillerato, por Medio de Entornos Virtuales de Aprendizaje, una Respuesta ante la Pandemia. conjunto de herramientas informáticas, que posibilitan la interacción didáctica. Mismo que posee cuatro características básicas:

a) Es un ambiente electrónico, no material en sentido físico, creado o constituido por tecnologías digitales.

b) Está hospedado en la red, misma a la que se puede acceder de manera remota a sus contenidos a través de algún tipo de dispositivo con conexión a internet.

c) Las aplicaciones o programas informáticos de que consta sirven de soporte para las actividades formativas tanto de docentes como de alumnos.

d) No se produce una relación didáctica en ellos, cara a cara, (como en la enseñanza presencial), sino mediada por tecnologías digitales, por ello los Entonos Virtuales de aprendizaje (EVA), permiten el desarrollo de acciones educativas sin necesidad de que tanto los docentes como alumnos coincidan en el espacio o en el tiempo.

Por su parte para García (2005, p.3), los entornos virtuales de aprendizaje, definen el sistema e-learning como una capacitación no presencial, con las plataformas tecnológicas, que posibilitan o flexibilizan el acceso y el tiempo en la enseñanza y aprendizaje, adecuándolos a las habilidades, necesidades o disponibilidades en cada participante, además garantizan ambientes de aprendizaje colaborativos mediante herramientas de comunicación síncrona o asíncrona, potenciando el proceso de gestión basado en competencias.

Por su parte Baez- Estradas y Ossandon (2015 p.844) refieren a Huang, Rauch \& Liaw, 2010,).y presentan a los Entornos Virtuales de aprendizaje (EVA), permiten un aprendizaje colaborativo basado, no tanto en una transmisión de conocimiento, sino en su construcción a través de interacción sociocultural, de forma que los individuos pasan a ser agentes activos en el proceso de aprendizaje, por lo tanto, no se limitan a absorber información, sino se conectan con el conocimiento previamente adquirido a fin de construir el suyo propio, en consecuencia el profesor y los contenidos que imparte, ya no son lo importante, ahora lo es, el alumno con su desarrollo en este sentido, los profesores se convierten en mediadores o catalizadores de este proceso de construcción y apropiación del conocimiento tal como lo menciona.
Por su parte, Cabero (2006, p.3), destaca como ventajas de los EVA los siguientes elementos: a) pone a disposición de los alumnos un amplio volumen de información, b) facilita la actualización de información y de contenidos, c) flexibiliza la información, independientemente del espacio o el tiempo en el cual se encuentren el profesor y el estudiante, d) permite la deslocalización del conocimiento, e) facilita la autonomía del estudiante; f) ofrece diferentes herramientas de comunicación sincrónica $\mathrm{y}$ asincrónica para los estudiantes $\mathrm{y}$ profesores, g) promueve una formación multimedia, grupal o colaborativa, entre otras. Evidencia al entorno virtual de aprendizaje, como un espacio común en línea, entre un grupo de personas que realizan tareas, gestiona, evalúan y reflexionan conocimientos, saberes y procedimientos, mediante un intercambio flexible.

Los entornos virtuales de aprendizaje son apropiados para crear y desarrollar programas de estudio de acuerdo con las necesidades de la institución y tecnologías a utilizar en plataformas de aprendizaje con herramientas educativas en la web, los cuales son espacios virtuales con accesos restringidos, sólo para los usuarios quienes responden a roles de docentes o alumnos. Por su parte Conalep, Nuevo León, dispone de herramientas tecnológicas para la oferta académica virtual, por medio de la plataforma Moodle, misma que garantizar una formación constante de sus estudiantes y docentes ante contingencias inesperadas como la pandemia de COVID-19. (Secretaría de Educación Pública, 2020).

La tecnología digital, presenta un cambio en nuestra sociedad, al incorporar los entornos virtuales de aprendizaje, se promueve la adquisición de nuevas habilidades mediante herramientas de comunicación, en la vida cotidiana, así como en el ámbito educativo. Como lo hacen notar (Burin, Coccimiglio, González \& Bulla, 2016, p.193) las nuevas tecnologías han cambiado la vida de las personas, exigiendo desarrollar una serie de habilidades digitales necesarias para acceder, comprender e interpretar el caudal de información al que se tiene acceso, tanto en la vida cotidiana como en ámbitos educativos o laborales.

Desde la perspectiva de Morales (2013), se destaca que, la formación del profesorado en TIC es una de las áreas prioritarias en la actual sociedad del conocimiento, al desempeñar un papel muy importante en las grandes líneas en las que se enmarcarán nuestros 
sistemas educativos en este nuevo siglo, que se centrarán en la innovación, la globalización, la ruptura de las fronteras culturales y lingüísticas, la movilidad virtual de los estudiantes, la emigración y la formación continua.

Por su parte, Organista, Sandoval, Mcanally \& Lavinge (2016) señalan que, la habilidad digital se concibe como el nivel de dominio que posee el individuo para manejar las Tecnologías de la Información y la Comunicación (TIC), la cual requiere de una práctica repetitiva de diversas tareas relacionadas con ejecuciones de tipo operativo e informacional. En el mismo sentido, Cuartas \& Quintero (2014, p.8), destacan que la alfabetización digital y las nuevas habilidades son necesarias para colaborar de forma digital y contribuir en el conocimiento colectivo y sostienen que el efecto de la tecnología va más allá de sólo alfabetizar.

En concordancia con los anterior, Rodríguez, Restrepo \& Aránzazu (2016) hacen referencia a Jadhav (2011) quien destaca la importancia de que las prácticas curriculares estimulen a los docentes, a utilizar las TIC en su práctica diaria, por medio de procesos acordes con las necesidades o políticas institucionales. En el mismo tenor el gobierno de México impulsa el programa @ aprende 2.0, con la finalidad de que todos los sectores sociales puedan aprovecharse o beneficiarse de las TIC, bajo este contexto, el programa de inclusión digital busca promover el desarrollo de habilidades digitales y el pensamiento computacional (México Digital, 2017).

De acuerdo con lo anterior, Conalep Nuevo León desde el 2013, impulsa una cultura digital, enfocada en capacitar y certificar las habilidades digitales de sus docentes usando en herramientas digitales que abarcan desde la alfabetización digital, así como aplicaciones de oficina, programas especializados, así como aplicaciones de diseño gráfico, diseño industrial, aplicaciones tecnológicas, por mencionar algunas, manteniendo una constante modernización docente.

Para Conalep Nuevo León, uno de los principales intereses institucionales, es que sus docentes adquieran las habilidades digitales $\mathbf{o}$ mejoren sus capacidades en la construcción de aulas virtuales, de esta manera, puedan contribuir a mejorar las experiencias de enseñanza aprendizaje en los entornos virtuales solicitados por la institución, se incita que los docentes sean transmisores de conocimiento al realizar una función de facilitadores y modeladores de habilidades tecnológicas en los estudiantes. El presente trabajo de investigación se estructura por los siguientes elementos: introducción, metodología, resultados, conclusiones por último las referencias bibliográficas.

\section{Metodología}

El diseño metodológico en el cual se fundamenta ésta investigación es bajo el enfoque cuantitativo, el cual, consiste en el contraste de teorías ya existentes a partir de una serie de hipótesis surgidas de la misma, siendo necesario obtener una muestra, ya sea en forma aleatoria o discriminada, pero representativa de una población o fenómeno objeto de estudio, (Tamayo, 2007, p.59), por lo tanto se circunscribe al enfoque cuantitativo pues parte de conceptos teóricos para desarrollar las habilidades digitales a una muestra específica de docentes de la institución, se considera exploratoria, al ser una primera aproximación al desarrollo del proceso de capacitación de docentes en habilidades digitales, en respuesta a las necesidades de salud actuales, así mismo es descriptiva pues da cuenta del proceso realizado con docentes en el CONALEP Nuevo León.

La población de estudio en esta investigación fue de 1,048 docentes contratados en el ciclo semestral febrero - julio de 2020, distribuidos en los 17 plantes del estado de Nuevo León. La muestra de docentes participantes, fue seleccionada por parte de la dirección académica, respondiendo a las necesidades de la institución, se delimitó a docentes quienes han demostrado tanto actitudes positivas como participativas hacia la institución, por lo tanto, se concretó por parte de la dirección académica, una participación de 66 docentes, que incluyen a 30 hombres así como 36 mujeres de los diferentes planteles del Conalep Nuevo León, cuyo rango de edad oscila entre los veinte y setenta años.

El proceso de recolección de datos se estableció para dar respuesta al objetivo planteado, por medio de la implementación de un curso en línea, el cual tuvo como meta guiar a los docentes en el desarrollo de sus habilidades digitales y aplicación de estas por medio de la creación e implementación de un aula virtual "prototipo" el cual sea modelo o pauta para el diseño de sus asignaturas.

Conalep Nuevo León, cuenta con su propio entorno virtual de aprendizaje, alojado en la dirección general estatal, dependencia que gestiona el servicio, presenta el 
Implementación de un Modelo de Capacitación para Docentes de Bachillerato, por Medio de Entornos Virtuales de Aprendizaje, una Respuesta ante la Pandemia. equipamiento, infraestructura y personal administrativo necesario para su funcionamiento, se encuentra disponible para los 17 planteles de la institución, se utilizó la herramienta de gestión de aprendizaje (Moodle) propia de Conalep Nuevo León, en la cual se diseñó el curso en línea siendo el instrumento para el desarrollo de esta investigación, a continuación, se describe el diseño e igualmente el desarrollo del curso.

El curso de capacitación a docentes para el desarrollo de habilidades digitales, se diseñó en respuesta a las necesidades del Conalep, Nuevo León, derivadas de la emergencia de salud pública, se denominó "Diseño de Cursos en Línea: Prototipo de un Aula Virtual", con una duración de 20 horas, distribuidas en 10 horas teóricas; las cuales se realizaron por medio de sesiones síncronas para favorecer el proceso de comunicación y comprensión de la información, así mismo, el seguimiento docente, adicionalmente se implementaron 10 horas prácticas, las cuales se realizaron de manera asíncrona, el curso se efectuó del 5 al 11 de mayo del 2020 en sesiones programadas, por medio un entorno virtual, en plataforma Moodle, el curso se distribuyó de acuerdo a los lineamientos de la dirección académica en un periodo de 5 días.

Las actividades a desarrollar por parte de los docentes se dividieron en aspectos tanto teóricos como prácticos, así mismo se realizó el proceso de verificación de aprendizajes por medio de instrumentos de evaluación, definidos para cada contenido temático, el mínimo aprobatorio establecido por parte de la dirección académica, fue de ocho puntos generales por parte de los docentes, se incluyó un foro dentro del entorno virtual, para favorecer el proceso de comunicación e interacción entre los docentes participantes en el curso. Una vez desarrollado e implementado el curso de capacitación en habilidades digitales, se optó por analizar los datos obtenidos de manera cuantitativa por medio de la aplicación de herramientas estadísticas básicas, en consecuencia, se pudo medir, así como verificar el aprendizaje por parte de los docentes, en los aspectos tanto teóricos como de aplicación.

\section{Resultados}

El proceso de capacitación en habilidades digitales implementado para los docentes de Conalep
Nuevo León se impartió a una muestra compuesta por 66 profesores, los cuales, desarrollaron actividades tanto teóricas cómo prácticas, conforme al objetivo definido, el mínimo aprobatorio se determinó en 8 puntos, por parte del área de coordinación académica e instructores del curso. Los resultados se presentan de acuerdo con los datos obtenidos, respondiendo al objetivo general de esta investigación, el cual consistió en implementar un modelo de capacitación a docentes, en modalidad en línea, para adquirir conocimientos en habilidades digitales en el desarrollo y construcción de un aula virtual.

Se describe la información obtenida en cada uno de los 5 módulos del curso, se presentan las necesidades detectadas en los docentes, así como los elementos obtenidos por género y rango de edad, los cuales se establecen en 2 rubros, componentes teóricos y componentes de ejecución los cuales conforman el contenido desarrollado en el proceso de capacitación. Durante el desarrollo del curso, 2 docentes por motivos personales, no continuaron en el programa de capacitación, constituyendo la muestra en 64 docentes; de este número aprobaron el curso 58 profesores, al acreditar los contenidos teóricos y actividades prácticas, y obtener un promedio de 8 puntos mínimo entre ambos rubros, lo cual representa un $88 \%$ de aprobación respecto al total, de manera complementaria hubieron 6 profesores quienes no lograron acreditar el curso, al no obtener el puntaje mínimo definido.

Los resultados se presentan en función de los rubros del curso, tanto teóricos como prácticos, participaron activamente los 64 docentes, en ambos elementos del proceso de capacitación, en un primer momento se desarrollaron todos los contenidos teóricos y posteriormente se realizaron los contenidos prácticos; en el módulo uno (1), se dio a conocer tanto los conceptos como propósitos de los entornos virtuales de aprendizaje, así mismo, el identificar los elementos de un ambiente de aprendizaje, los modelos de aprendizaje y su diferencia del e-learning, b-Learning y m-Learning. En este módulo se obtuvo un resultado $100 \%$ de aprobación.

En el módulo dos (2), se dio a conocer las competencias del rol de un tutor o facilitador, los enfoques específicos dentro de la enseñanza directa, las cualidades y habilidades para los entornos virtuales de aprendizaje, este módulo se tuvo un 
Implementación de un Modelo de Capacitación para Docentes de Bachillerato, por Medio de Entornos Virtuales de Aprendizaje, una Respuesta ante la Pandemia. registro de aprobación de 89\%. Tanto los elementos de las plataformas y los EVA se abordaron en el módulo tres (3), de igual manera se revisó la relevancia de los contenidos como recursos didácticos y actividades, en este módulo la aprobación por parte de los docentes fue del 97\%; el módulo cuatro (4), se enfocó en las características de una educación tradicional a diferencia de un tutor o facilitador para entornos virtuales de aprendizaje, con una aprobación del 92\%. Para finalizar en el módulo cinco (5), se obtuvo una aprobación del $73 \%$, se estableció en el desarrollo de una estructura organizada con el diseño instruccional acorde a las necesidades educativas con el fin de lograr una transmisión de conocimiento, consolidando un aula virtual.

Respecto a los resultados generales de los contenidos teóricos, se identifican lo pertinente al desarrollo y diseño instruccional, como el aspecto que requiere reforzamiento por parte de los docentes, pues se obtuvo una aprobación más baja, este elemento teórico es pertinente para consolidar el desempeño de los docentes en EVA. Para complementar y considerando los grupos de edad presentes dentro de la muestra, los cuales abarcan de los 20 a los 70 años de edad, se realizó un análisis para identificar los resultados obtenidos, y sí estos arrojaban diferencias por edad.

A continuación, en la tabla uno (1), se presentan los resultados por rango de edad.

\section{Tabla 1}

Resultados por rango de edad y aprobación teórica del módulo.

\begin{tabular}{cccccc}
\hline $\begin{array}{c}\text { Rango de } \\
\text { edad }\end{array}$ & $\begin{array}{c}\text { Módulo } \\
\mathbf{1}\end{array}$ & $\begin{array}{c}\text { Módulo } \\
\mathbf{2}\end{array}$ & $\begin{array}{c}\text { Módulo } \\
\mathbf{3}\end{array}$ & $\begin{array}{c}\text { Módulo } \\
\mathbf{4}\end{array}$ & $\begin{array}{c}\text { Módulo } \\
\mathbf{5}\end{array}$ \\
\hline $\mathbf{2 0}$ a 30 & 19 & 9 & 9 & 19 & 17 \\
\hline $\mathbf{3 1}$ a 40 & 15 & 3 & 5 & 14 & 10 \\
\hline $\mathbf{4 1}$ a 50 & 13 & 0 & 1 & 11 & 7 \\
\hline $\mathbf{5 1}$ a 60 & 12 & 0 & 2 & 11 & 10 \\
\hline $\mathbf{6 1}$ a 70 & 5 & 5 & 5 & 4 & 3 \\
\hline Total & $\mathbf{6 4}$ & $\mathbf{5 7}$ & $\mathbf{6 2}$ & $\mathbf{5 9}$ & $\mathbf{4 7}$ \\
\hline Porcentaje & $\mathbf{1 0 0 \%}$ & $\mathbf{8 9 \%}$ & $\mathbf{9 7 \%}$ & $\mathbf{9 2 \%}$ & $\mathbf{7 3 \%}$ \\
\hline
\end{tabular}

Nota. Esta tabla muestra la relación entre rango de edad y aprobación en el aspecto teórico. Elaboración propia.

En el análisis de los resultados obtenidos por módulo, se identifican los módulos dos (2) y cinco (5) cómo los módulos con menor aprobación, pues obtuvieron un puntaje de entre $89 \%$ y $73 \%$ respectivamente, dentro de éstos, los grupos de edad entre 31-40 años, así como el de 61- 70 años, son los rangos que presentan el menor puntaje de aprobación, por lo tanto, se evidencia un pertinente reforzamiento en estos elementos teóricos, en los grupos de edad especificados.

El segundo elemento componente del curso, fue el práctico o de ejecución, en el cual los docentes debían aplicar el material teórico incluido en el aula virtual. el curso constó de cinco (5) prácticas, a partir de las cuales se obtuvo un resultado general de aprobación del $85 \%$.En la práctica uno (1), se les solicitó diseñar imágenes de los títulos de cada unidad de aprendizaje, delimitadas por el programa de estudios o asignatura a organizar por secciones, mismo que obtuvo un resultado de $86 \%$ de aprobación, dicho puntaje fue repetitivo y consistente en la práctica dos (2), que tuvo como objetivo, diseñar imágenes de botones a utilizar en cada sección del plan de estudios o asignatura a organizar por secciones.

Seguidamente en la práctica tres (3), referente a diseñar imágenes para uso académico, de manera complementaria se reconocen las prácticas sobre diseño instruccional y diseño de bloque, estas actividades resultan ser de menor aprobación, en consecuencia, se establecen como elementos de ejecución necesarios a reforzar. En el análisis por grupos de edad en las prácticas del curso, se confirma lo referente a las prácticas cuatro (4) y cinco (5) cómo los elementos necesarios de fortalecer en el aprendizaje de los docentes, al obtener menor aprobación, lo anterior se presenta en la tabla 2.

\section{Tabla 2}

Resultados por rango de edad y prácticas.

\begin{tabular}{cccccc}
\hline $\begin{array}{c}\text { Rango de } \\
\text { Edad }\end{array}$ & $\begin{array}{c}\text { Práctica } \\
\mathbf{1}\end{array}$ & $\begin{array}{c}\text { Práctica } \\
\mathbf{2}\end{array}$ & $\begin{array}{c}\text { Práctica } \\
\mathbf{3}\end{array}$ & $\begin{array}{c}\text { Práctica } \\
\mathbf{4}\end{array}$ & $\begin{array}{c}\text { Práctica } \\
\mathbf{5}\end{array}$ \\
\hline 20 a 30 & 18 & 18 & 18 & 16 & 17 \\
31 a 40 & 13 & 14 & 13 & 12 & 13 \\
41 a 50 & 10 & 10 & 10 & 11 & 11 \\
51 a 60 & 11 & 10 & 11 & 10 & 9 \\
61 a 70 & 3 & 3 & 3 & 4 & 4 \\
\hline Total & 55 & 55 & 55 & 53 & 54 \\
\hline
\end{tabular}

Nota. Esta tabla muestra la relación entre rango de edad y aprobación en el aspecto práctico. Elaboración propia.

De manera adicional a las aspectos teóricos y prácticos del curso de capacitación, se tenía alojado un foro de intercambio grupal, en el cual los docentes podían comunicarse entre ellos, la información presentada en el foro, evidencia al grupo de edad 61 
Implementación de un Modelo de Capacitación para Docentes de Bachillerato, por Medio de Entornos Virtuales de Aprendizaje, una Respuesta ante la Pandemia. a 70 años, como aquellos con más inquietudes al momento de trabajar en el aula virtual. Los datos obtenidos en el foro permitieron identificar las necesidades y vacíos de conocimiento por parte de los docentes, referentes al proceso de ejecución de las prácticas cuatro (4) y cinco (5), se logra identificar "inseguridad" al realizar las actividades en el aula virtual, refieren la comprobación de las acciones a seguir, así como la verificación de la información.

Lo anterior concuerda con lo desarrollado por Cabero (2004), la falta de formación didácticopedagógica de las tecnologías es uno de los mayores obstáculos para los profesores en los procesos educativos. Ante la pandemia y en virtud de los lineamientos de salud pública definidos por parte del gobierno mexicano, la respuesta institucional se realizó por medio de la implementación del curso de capacitación para el desarrollo de las habilidades digitales, constituyendo un elemento de impacto positivo en la comunidad docente, y permitiendo la incorporación de tecnología por medio de un entorno virtual de aprendizaje, obteniendo una respuesta asertiva, ante la solicitud no sólo de distanciamiento social sino también de reducción de movilidad ante el COVID-19.

Los resultados cuantitativos arrojan como evidencia de la consecución del objetivo, logrando el desarrollo de las habilidades digitales en el $88 \%$ de los docentes inscritos, se otorgan también estrategias efectivas de trabajo en línea, se consolidan conocimientos teóricos y prácticos y se evidencia en los docentes una necesidad de reforzar algunos elementos con el fin de consolidar la aplicación y ejecución de las aulas virtuales, resulta necesario, continuar con el desarrollo de las habilidades digitales por parte para de los docentes, mismo que se verá reflejado en la calidad ofrecida en los servicios educativos de Conalep Nuevo León.

\section{Conclusiones}

Ante la pandemia declarada a nivel mundial derivada del COVID-19 y el inminente cierre de las instituciones educativas, Conalep Nuevo León, presentó la implementación de manera emergente de las TIC en el contexto de los 17 planteles que lo conforman, era urgente dar respuesta a esta nueva normalidad, en consecuencia, se establece un plan de contingencia, enfocado en capacitar de manera inmediata a sus docentes para el desarrollo e implementación del aula virtual y en consecuencia de sus habilidades digitales.

Se adaptaron los métodos de enseñanza tradicionales al uso de tecnologías en entornos virtuales de aprendizaje, se propició la adquisición de conocimientos en habilidades digitales para el desarrollo y construcción de aulas virtuales, en donde se alojan los contenidos didácticos de cada una de las asignaturas de los programas, también se facilitó la interacción entre docentes y alumnos, por lo cual se dio continuidad al proceso de enseñanza, con la inserción a la modalidad a distancia, estos elementos permitieron una adaptación de contenidos didácticos así como la realización de evaluaciones en formato en línea.

A través del diseño, desarrollo e implementación de la capacitación a docentes en aulas virtuales, la institución satisface las necesidades y adecuaciones requeridas por la emergencia sanitaria y establece los lineamientos para brindar servicios de calidad, por medio del nuevo rol del docente, consolidándolo como facilitador en entornos virtuales de aprendizaje, y promoviendo la aplicación de habilidades digitales en su desempeño profesional.

El objetivo del trabajo fue el implementar un curso de capacitación para el desarrollo de las habilidades digitales de los participantes, el curso obtuvo una aprobación del $88 \%$, se dividió en aspectos teóricos y prácticos, con los resultados obtenidos se identifica el área de ejecución en el diseño instruccional, como el elemento con más demanda y atención por parte de los docentes, lo anterior concuerda con lo referido por Mayorga (2020 p.10) donde puntualiza que los docentes presentan deficiencias en los aspectos teóricos, de aplicación e intervención en el uso de las TIC, pero sobre todo en el diseño de ambientes virtuales de aprendizaje, en éste sentido se identifica al grupo de mayor edad (61-70 años) como el rango que presenta sus dudas de manera más constante en el desarrollo del curso.

La información obtenida evidencia el interés y disposición de docentes para seguir formándose y capacitándose en lo referente a la educación en línea, así mismo refieren la necesidad de más cursos formativos en educación en línea. La implementación del modelo de capacitación a docentes del Conalep Nuevo León constituye un hito, en los procesos formativos a docentes de la 
Implementación de un Modelo de Capacitación para Docentes de Bachillerato, por Medio de Entornos Virtuales de Aprendizaje, una Respuesta ante la Pandemia. institución, estableciéndola como una institución comprometida con la comunidad educativa y dispuesta a implementar procesos de mejorar y capacitación docente.

En concordancia con los resultados positivos obtenidos por parte de los docentes, la institución a través de la dirección académica estableció el curso de capacitación descrito en la presente investigación referente a entornos virtuales de aprendizaje, en una segunda edición dirigida a los 1,048 docentes pertenecientes a los 17 plantes del estado de Nuevo León, es decir, se convocó a la totalidad de docentes de la institución, esto establece medios de respuesta a las demandas derivadas de la pandemia, continuar los procesos educativos y alinear el desempeño de los docentes, es una oportunidad para mostrar que los procesos educativos no se detienen, sino al contrario, se fortalecen, preparando al personal académico con competencias en entornos virtuales.

\section{Referencias}

Arras, A., Valencia, A., \& Tejedor, F. (2014). Apertura. UDG Virtual. . Obtenido de Perspectiva de estudiantes de posgrado sobre escenarios de aprendizaje, condiciones de la docencia y competencias en TIC en las modalidades presencial y virtual: https://dialnet.unirioja.es/servlet/articulo?codigo $=5547080$

Baez-Estradas, M., \& Ossandón-Núñez, Y. (2015). Propiedades psicométricas de la escala de presencia social de las mentes en red y su aplicación en el Entorno Virtual de Enseñanza Aprendizaje UTAmed. Universitas Psychologica, 14(3), 843854. http://dx.doi.org/10.11144/Javeriana.upsy14-3.ppep

Betegon, L., Fossas, M., Martinez, E., \& y Ramos, M. (2010). Entornos virtuales como apoyo a la docencia universitaria presencial. Anuario jurídico y económico escurialense, 273 302

Burin, D., Coccimiglio, Y., González, F., \& Bulla, J. (mayo de 2016). Desarrollos recientes sobre habilidades digitales y comprensión lectora en entornos digitales. Psicología Conocimiento y Sociedad 6(1), 191-206. Obtenido de http://www.scielo.edu.uy/scielo.php?pid=S1688$70262016000100009 \&$ script $=$ sci_arttext\&tlng=pt

Cabero, J. (2004). Formación del profesorado en TIC. El gran caballo de batalla. Comunicación y Pedagogía. Tecnología y Recursos Didácticos, 195., 27-31.

Cabero, J. (2006). Bases pedagógicas del e-learning. Obtenido de Revista de Universidad y Sociedad del Conocimiento (RUSC). Vol. 3, nro $1 . \quad$ UOC.: https://ddd.uab.cat/pub/dim/16993748n6/16993748n6a4.pdf
Conalep. (29 de julio de 2011). Criterios generales aplicables al personal académico del CONALEP. Obtenido de Colegio Nacional de Educación Profesional Técnica: http://www.conalep.edu.mx/gobmx/normateca/Paginas/Servici os/Formacion-academica/05-EDITABLE-15112012CRITERIOSDOCENTES-2012-.pdf

Cuartas, M., \& Quintero, V. (2014). Congreso Iberoamericano de Ciencia, Tecnología, Innovación y Educación. Obtenido de Formación docente en el desarrollo de competencias digitales e informacionales a través del modelo enriquecido TPACK*CTS*ABP. ISBN:978-84-7666-210-6-Artículo 784, $1-20$ :

https://www.google.com/url?sa=t\&rct=j\&q=\&esrc=s\&source $=$ web $\& \mathrm{~cd}=\& \mathrm{ved}=2 \mathrm{ahUKEwjwkZPkq}$ nqAhVS5awKHWrgBY0QFjAAegQIBRAB\&url=https\%3A $\% 2 \mathrm{~F} \% 2 \mathrm{Fwww}$.oei.es\%2Fhistorico\%2Fcongreso2014\%2Fme moriactei\%2F784.pdf\&usg=AOvVaw0tFGE6GwNrS5h9Aw G-x9MQ

Departamento de comunicación global. (2020). UN, Naciones Unidas Covid - 19 Respuesta. Obtenido de La ONU ayuda a los más jóvenes a continuar sus estudios: https://www.un.org/es/coronavirus/articles/educaci\%C3\%B3n -covid-19-onu-ayuda-ni\%C3\%B1os-estudios-aprendizaje

DOF, D. O. (31 de marzo de 2020). ACUERDO ACTPUB/25/03/2020.14 SE MODIFICA Y ADICIONA EL DIVERSO ACT-EXT-PUB/20/03/2020.02. Obtenido de https://www.dof.gob.mx/nota_detalle.php?codigo $=5590793 \& \mathrm{f}$ echa $=31 / 03 / 2020 \&$ print $=$ true

García, F. (2005). Estado actual de los sistemas e-learning. Teoría de la Educación. Educación y Cultura en la Sociedad de la Información Educación y Cultura en la Sociedad de la Información, ISSN Electrónico:1138-9737.

Ibarra, C. (01 de noviembre de 2019). Secretaría Académica: Dirección de Formación Académica. Obtenido de Colegio Nacional de Educación Profesional Técnica. Gobierno de México.: https://www.gob.mx/conalep/acciones-y-programas/docentes28414

Landázuri, M. (13 de Enero de 2017). Repositorio Dspace. Obtenido de Tecnologías de información y comunicación que ayudan a mejorar el aprendizaje teórico práctico musical en la guitarra eléctrica dentro de niveles avanzados de educación superior.: http://dspace.uhemisferios.edu.ec:8080/xmlui/handle/1234567 $89 / 519$

Mayorga, M. (2020). Conocimiento, Aplicación e Integración de las TIC - TAC y TEP por los Docentes Universitarios de la Ciudad de Ambato. Revista Tecnológica-Educativa Docentes 2.0, 9(1), 5-11. doi:DOI: https://doi.org/10.37843/rted.v9i1.101

México Digital. (09 de febrero de 2017). Gobierno de México. Obtenido de Inclusión y habilidades digitales.: https://www.gob.mx/mexicodigital/articulos/inclusion-yhabilidades-digitales

Morales A, V. (2013). Desarrollo de competencias digitales docentes en la educación básica. Recuperado el 25 de julio de 2020, de Apertura, 5(1),88-97. ISSN: 1665-6180.: https://www.redalyc.org/articulo.oa?id=688/68830443008 
Implementación de un Modelo de Capacitación para Docentes de Bachillerato, por Medio de Entornos Virtuales de Aprendizaje, una Respuesta ante la

Organista, J., Sandoval, M., McAnally, L., \& Lavigne, G. (31 de Octubre de 2016). Revista Electrónica de Tecnología Educativa, doi:https://doi.org/10.21556/edutec.2016.57.673

Edutec.

Rodríguez, H., Restrepo, L., \& Aranzazu, D. (2016.). Redalyc, Sophia. Obtenido de Desarrollo de habilidades digitales docentes para implementar ambientes virtuales de aprendizaje en la docencia universitaria. Num.2, 261-270.: https://www.redalyc.org/pdf/4137/413746578009.pdf

Salinas, M. (1 de Abril de 2011). Entornos virtuales de aprendizaje en la escuela: tipos, modelo didáctico y rol del docente. Obtenido de Pontificia Universidad Católica Argentina: http://www.uca.edu.ar/uca/common/grupo82/files/educacionEVA-en-la-escuela_web-Depto.pdf

Secretaría de Educación Pública, S. C. (08 de abril de 2020). Boletín No. 93 Refuerza CONALEP Programa de Educación a Distancia por COVID-19. Garantiza la formación constante de sus estudiantes y docentes durante contingencia. Obtenido de Gobierno de México: https://www.gob.mx/sep/es/articulos/boletin-no-93-refuerzaconalep-programa-de-educacion-a-distancia-por-covid19?idiom=es

Secretaria de Educación Pública. (20 de abril de 2020). Boletín No. 93 Refuerza CONALEP Programa de Educación a Distancia por COVID-19. Obtenido de Garantiza la formación constante de sus estudiantes y docentes durante la contingencia.: https://www.gob.mx/sep/es/articulos/boletin-no-93-refuerzaconalep-programa-de-educacion-a-distancia-por-covid19? idiom=es

Secretaria de Gobernación . (10 de abril de 2020). Acuerdos por contingencia sanitaria están actualizados en el Diario Oficial de la Federación. Obtenido de Información importante Coronavirus COVID-19: https://www.gob.mx/segob/prensa/acuerdos-por-contingenciasanitaria-estan-actualizados-en-el-diario-oficial-de-lafederacion

Silva, J. (Marzo de 2004). EL Rol Moderador del Tutor en la Conferencia Mediada por Computador. Obtenido de Edutec: Revista Electrónica: https://www.edutec.es/revista/index.php/edutece/article/view/531/265

Tamayo, M. (2007). El proceso de la investigación científica. México: Limusa.

UNESCO. (2020). Coalición Mundial para la Educación. . Obtenido de Organización de las Naciones Unidas para la Educación, la Ciencia y la cultura: https://es.unesco.org/covid19/globaleducationcoalition

UNISEF, WHO, \& IFRC. (March de 2020). COVID-19: la FICR, UNICEF y la OMS publican una guía para proteger a los niños y apoyar la seguridad en las operaciones escolares. Obtenido de Organización Mundial de la Salud.: https://www.unicef.org/media/65716/file/Key\%20Messages\% 20 and $\% 20$ Actions $\% 20$ for $\% 20$ COVID-

$19 \% 20$ Prevention\%20and\%20Control\%20in\%20Schools_Ma rch\%202020.pdf 